\title{
The first application of epiluminescence dermoscopy in erythema nodosum
}

\section{Antonio Chuh', Vijay Zawar', Regina Fölster-Holst ${ }^{3}$}

\author{
${ }^{1}$ Department of Family Medicine and Primary Care, The University of Hong Kong and Queen Mary Hospital, Hong Kong, \\ ${ }^{2}$ Department of Dermatology, Godavari Foundation Medical College and Research Center, DUPMCJ, India, \\ ${ }^{3}$ Universitätsklinikum Schleswig-Holstein, Campus Kiel, Dermatologie, Venerologie und Allergologie, Germany
}

Corresponding author: Dr Antonio Chuh, E-mail: antonio.chuh@yahoo.com.hk

\begin{abstract}
We reported an adult female with a clinical diagnosis of erythema nodosum. The patient declined lesional biopsy. We applied epiluminescence dermoscopy, which revealed features compatible with panniculitis. We managed conservatively. The rash remitted four weeks since rash onset, leaving only post-inflammatory hyperpigmentation. Dermoscopic examination cannot replace lesional biopsy for histopathology for a diagnosis of erythema nodosum to be properly confirmed. However, there are patients with clinical diagnoses of erythema nodosum who would not give consent for lesional biopsies, and patients presenting to dermatologists when the rash is already remitting. We thus described the dermoscopic findings of our patient in this report. The applicability of dermoscopy to patients with erythema nodosum and differential diagnoses of such is yet to be evaluated by further studies.
\end{abstract}

Key words: Contact dermoscopy; Cross-polarisation; Dermatoscope; Dermoscope; Digital epiluminescence dermoscope; Polarised light

\section{INTRODUCTION}

Epiluminescence dermoscopy (ED) is increasingly being applied to dermatological conditions other than cutaneous malignancies. We present here the first application of ED on a patient with erythema nodosum (EN).

\section{CASE REPORT}

A 32-year-old lady consulted us for more than two weeks' history of painful skin lesions over her lower limbs. She was also enduring frequent productive cough with purulent sputum. Her appetite was fair, but she reported no recent weight loss. She had no monoarthralgia and no polyarthralgia. She enjoyed good past health. Travel, contact, sex, and drug histories were unremarkable.
Our examination revealed a well and afebrile patient with no pallor and no jaundice. Multiple plaques were noted on the lower aspect of her thighs, latero-posterior and posterior aspects of her legs, and both ankles (Fig. 1). The lesions were discrete, erythematous to purple-coloured, subcutaneous, and slightly elevated. They were monomorphic but differed in sizes from $0.5 \mathrm{~cm}$ to $4 \mathrm{~cm}$. The margins of lesions were not clearly demarcated. The lesions were not blanchable. All other skin surfaces including the external genitalia were uninvolved.

Her mucosal surfaces were unaffected. Her throat was inflammed, with tonsils being enlarged. No exudate was noted. There was no inguinal or other lymphadenopathy. Examination of her cardiovascular system, chest, abdomen, and musculoskeletal system revealed no abnormality otherwise.

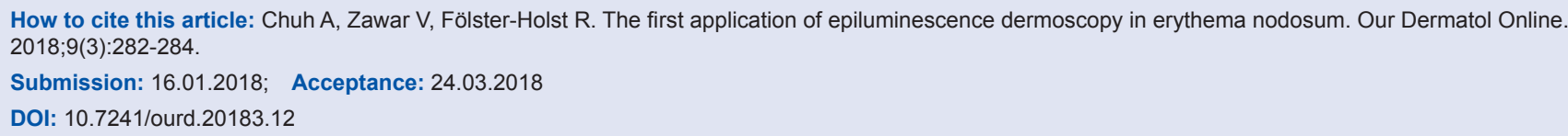




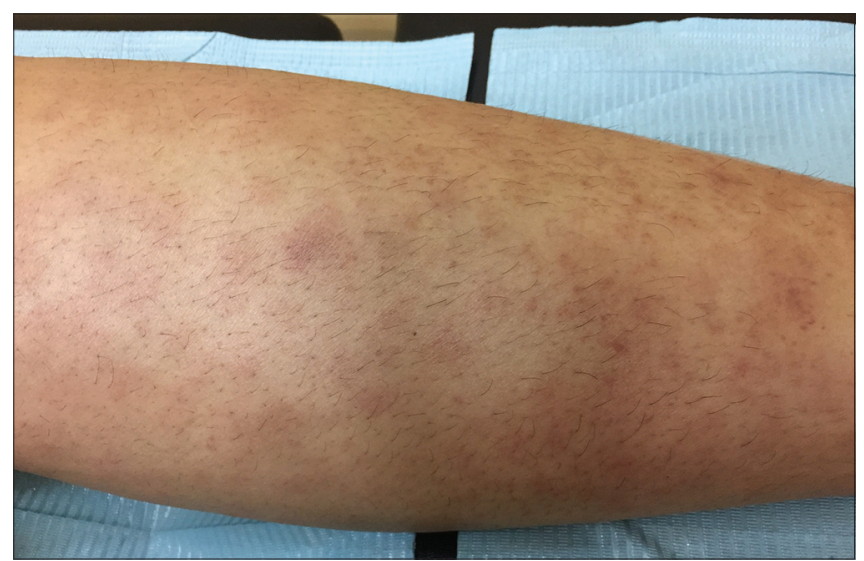

Figure 1: Multiple discrete purple-coloured macules on the anterior aspects of both legs of a patient with a clinical diagnosis of erythema nodosum.

Dermoscopic examination without cross-polarisation (Fig. 2a) revealed erythema in the lesional skin (centre in the figure) as compared to the normal skin (peripheral in the figure). The skin creases were largely uninterrupted from non-lesional to lesional surfaces. Examination under DED revealed one entire lesion comprising eight to nine lobules (Fig. 2b), compatible with the histopathology in EN. The separations between the lobules would correspond to inter-lobular septa in EN. Based on the setting of our ED device, we should be viewing features just beneath the dermis. This was also compatible with the depth of panniculitis in $\mathrm{EN}$.

Results for her complete blood count, random glucose, liver function tests, and renal function tests were normal. ESR was elevated to $64 \mathrm{~mm}$ in the first hour. CRP was elevated to $98.4 \mathrm{mg} / \mathrm{l}$ (reference: less than $5 \mathrm{mg} / \mathrm{l})$. Qualitative anti-streptolycinO-titre was positive. Monospot test and serology against Mycoplasma spp were negative. Antinuclear autoantibodies and rheumatoid factor were negative. Chest X-ray revealed no evidence of acute chest infection, tuberculosis, or sarcoidosis. The patient declined a deep lesional punch biopsy.

We believed that the clinical findings and the findings in $\mathrm{ED}$ were adequate for making a diagnosis of $\mathrm{EN}$, with streptococcal infection being a possible triggering factor. We prescribed oral cefuroxime $250 \mathrm{mg}$ twice daily for one week, and oral diclofenic sodium $25 \mathrm{mg}$ twice daily as necessary for the relief of pain. We also recommended compression hosiery. The patient attended us again two weeks later. Almost all skin lesions had remitted, leaving post-inflammatory hyperpigmentation only.

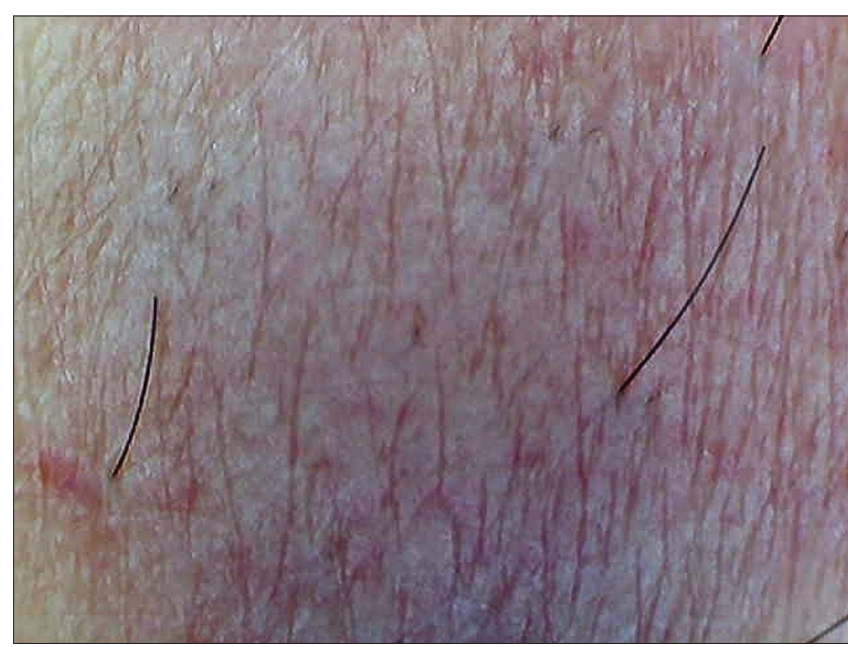

Figure 2a: Digital dermoscopic image with no cross-polarisation demonstrating one lesion. Erythema was noted. The skin creases were not interrupted. Apart from such, no additional information was provided.

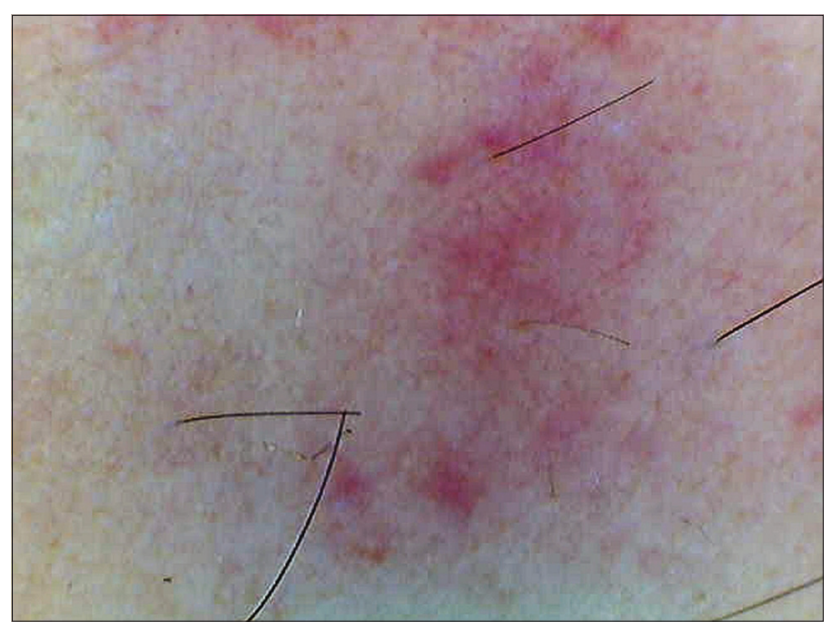

Figure 2b: Digital epiluminescence dermoscopy with the highest level of polarisation, showing the layer just deeper than the dermis. One entire lesion was depicted, composing of around eight erythematous lobules. These lobules substantiated the presence of lobular panniculitides. The separations between the lobules could represent the inter-lobular septa in erythema nodosum. Swollen blood vessels were noted, with no telangiectasia. Overall, these features were compatible with known features in erythema nodosum.

\section{DISCUSSION}

The configuration shown Figure $2 \mathrm{~b}$ is spectacular. To our best knowledge, images akin to this figure has not been published. This was why we elected to submit this patient report despite our having findings on only one patient. However, we do not believe that our findings can be generalised to other patients, nor do we advocate dermoscopy replacing lesional histopathology as the proper substantiation in diagnosing EN.

Making a proper diagnosis of $\mathrm{EN}$ is important. First and foremost, underlying causes including 
pulmonary tuberculosis, other infectious diseases, autoimmune diseases, malignancies, and drugs must be excluded [1-3]. Secondly, although EN is usually a self-limiting disease remitting spontaneously in six to eight weeks, the pain can affect the mobility of patients, thus affecting their schoolings or works. Thirdly, some patients will keep on developing crops of lesions, thereby progressing to chronic EN.

The management for EN would be to treat the underlying cause if identifiable. Otherwise, for recalcitrant $\mathrm{EN}$, systemic corticosteroids might be indicated. However, one important differential diagnosis of EN is cellulitis. The bacterial infection in cellulitis will flare up if a wrong diagnosis of $\mathrm{EN}$ is made and systemic corticosteroids are administered.

EN is a panniculitis caused by delayed hypersensitivity responses to antigens. It belongs to the group of panniculitis which are septocentric and without vasculitis $[4,5]$. The histopathological changes of EN are those of septal and lobular panniculitis [4-6]. Septa would divide fat lobules. Perivascular and periadnexal lymphocytic, neutrophilic, and eosinophilic infiltrates are usually present.

Inspection with naked eyes or with a magnifying glass might not be adequate to explore the configuration of EN owing to (i) the skin creases distracting the view; (ii) the tissue changes being too deep; and (iii) the changes being too small for the visual acuity.

These directly echo the roles of ED. Against (i), crosspolarisation in ED ablates reflections from the skin service, rendering the skin creases invisible. Against (ii), some dermoscopes incorporate settings for adjusting the extent of cross-polarisation, thus allowing visualisation of the deep layers of affected fatty tissues. Against (iii), ED would magnify the images optically and digitally so that small details can be displayed.
Deep lesional biopsy is painful, can cause complications, and consumes time and expenses. For patients with a provisional diagnosis of EN but, like our patient, decline lesional biopsy, the application of ED might therefore substantiate the diagnosis to some extent. Moreover, there are patients with suspected EN whose rashes have already been largely remitted by the time they are referred to see a dermatologist. The need for lesional biopsy might also be lessened in such clinical scenarios.

However, our knowledge of dermoscopic changes in $\mathrm{EN}$ and important differential diagnoses of $\mathrm{EN}$ is still premature. Further studies are necessary to investigate the validity and reliability of applying ED in patients with $\mathrm{EN}$ or differential diagnoses of $\mathrm{EN}$.

\section{CONSENT}

The examination of the patient was conducted according to the Declaration of Helsinki principles.

\section{REFERENCES}

1. Babamahmoudi F, Amuzgar A, Mousavi T, Davoodi L. Erythema nodosum: what should we consider about it? Caspian J Intern Med. 2016;7:304-5.

2. Dogan S, Karaduman A, Evans SE. Clinical and laboratory characteristics of patients with erythema nodosum. Skinmed. 2016;14:99-103.

3. Chowaniec M, Starba A, Wiland P. Erythema nodosum - a review of the literature. Reumatologia. 2016;54:79-82.

4. Thurber S, Kohler S. Histopathologic spectrum of erythema nodosum. J Cutan Pathol. 2006;33:18-26.

5. Wick MR. Panniculitis: a summary. Semin Diagn Pathol. 2017;34:261-72.

6. Böer-Auer A. Differential diagnosis of panniculitides. Hautarzt. 2016;67:555-69.

Copyright by Antonio Chuh, et al. This is an open-access article distributed under the terms of the Creative Commons Attribution License, which permits unrestricted use, distribution, and reproduction in any medium, provided the original author and source are credited.

Source of Support: Nil, Conflict of Interest: None declared. 